\title{
Erectile Dysfunction in Diabetic Patients: From Etiology to Management
}

\author{
Rossella Cannarella * ${ }^{\circ}$, Federica Barbagallo, Rosita A. Condorelli $\mathbb{1}$, Carmelo Gusmano, Andrea Crafa, \\ Sandro La Vignera (1) and Aldo E. Calogero (1)
}

Citation: Cannarella, R.; Barbagallo, F.; Condorelli, R.A.; Gusmano, C.; Crafa, A.; La Vignera, S.; Calogero, A.E. Erectile Dysfunction in Diabetic Patients: From Etiology to Management. Diabetology 2021, 2, 157-164. https://doi.org/10.3390/ diabetology2030014

Academic Editor: Peter Clifton

Received: 29 March 2021

Accepted: 17 August 2021

Published: 4 September 2021

Publisher's Note: MDPI stays neutral with regard to jurisdictional claims in published maps and institutional affiliations.

Copyright: (C) 2021 by the authors Licensee MDPI, Basel, Switzerland. This article is an open access article distributed under the terms and conditions of the Creative Commons Attribution (CC BY) license (https:/ / creativecommons.org/licenses/by/ $4.0 /)$.
Department of Clinical and Experimental Medicine, University of Catania, 95123 Catania, Italy; federica.barbagallo11@gmail.com (F.B.); rosita.condorelli@unict.it (R.A.C.); carmelo.gusmano@yahoo.it (C.G.); crafa.andrea@outlook.it (A.C.); sandrolavignera@unict.it (S.L.V.); aldo.calogero@unict.it (A.E.C.)

* Correspondence: rossella.cannarella@phd.unict.it

\begin{abstract}
Diabetes mellitus (DM) is a widespread chronic disease with a prevalence that is expected to further increase in the near future. The classical management of DM includes the normalization of the glycometabolic profile and the evaluation of cardiac and cerebral vascular health by the intervention of an array of different specialists. However, so far, sexual dysfunctions are still a neglected complication in patients with DM, although there is an elevated prevalence of this longterm complication in male and female patients. Furthermore, some of them may represent a sign of vascular alteration and/or hypogonadism and require timely management to prevent the onset of major adverse cardiac events. This narrative review briefly summarizes the current evidence on epidemiology, pathogenesis, diagnosis, and therapy of erectile dysfunction in male patients with DM to support diabetologists in clinical practice.
\end{abstract}

Keywords: male sexual dysfunction; sexual health; sexual distress; diabetes; hyperglycemia

\section{Introduction}

Diabetes mellitus (DM) is a chronic and widespread disease, with a global prevalence of $9.3 \%$ in 2019 . Worryingly, its prevalence is expected to increase in the near future, by $25 \%$ in 2030 and by $51 \%$ in 2045 [1]. Several long-term complications are associated with DM, such as diabetic nephropathy, retinopathy, neuropathy, and micro- and macro-angiopathy. Sexual disorders represent one of the neglected long-term complications that can affect both male and female diabetic patients. In men, DM-related sexual dysfunction includes disorders of erection and ejaculation. Their timely diagnosis and proper management would allow not only the increase of the patients' wellbeing and quality of life but can positively impact the primary prevention of major adverse cardiac events (MACEs).

Therefore, this narrative review briefly summarizes the current knowledge of the relationship between sexual health and DM in men. Epidemiological evidence, pathogenesis, diagnosis, and therapy of sexual disorders, mainly focusing on erectile dysfunction (ED) in male diabetic patients, are discussed.

\section{Erectile Dysfunction}

ED is defined as the persistent failure to achieve or to maintain a penile erection satisfactory for sexual intercourse [1]. Penile erectile structures are the corpora cavernosa, which receive blood supplies by the internal pudendal artery that continues as the penile artery. Corpora cavernosa are made of sinusoids capable of notable volume expansion. Furthermore, they are enclosed in Buck's fascia which rigidly limits their expansion beyond a specific point and this allows the penis to reach its maximal rigidity [2].

A sequential series of events takes place during erection. In the flaccid state, cavernous smooth musculature shows a tonic contraction, allowing only minimal arterial flow for nutritional purposes. When sexual stimulation starts, neurotransmitters are released by the 
cavernous nerve terminals with an ensuing relaxation of cavernous smooth muscles. This leads to the dilation of the arterioles with a subsequent arterial blood flow increase. Sinusoids in turn expand and the subtunical venular plexus is compressed between sinusoids and the tunica albuginea, decreasing the venous outflow [2]. Erection depends upon the parasympathetic nervous system, which causes smooth muscle relaxation by nitric oxide (NO)-mediated action [2].

As known from physiology, the integrity of both vascular and nervous systems is needed for penile erection. Furthermore, NO-mediated erection is dependent on testosterone (T) serum levels [3]. Therefore, the etiology of ED includes psychogenic (e.g., due to stress, depression, or anxiety [4]) and organic forms. The former in turn comprises endocrine causes, such as hypogonadism, hyperprolactinemia, hyper- or hypothyroidism, Cushing's syndrome, Addison's disease, and non-endocrine causes, such as vasculogenic, neurogenic, and/or iatrogenic forms [2].

The vasculogenic etiology includes all the disorders that affect the arterial penile flow (e.g., atherosclerosis, ischemic heart disease, and peripheral vascular disease) and/or the venous outflow (such as venous incontinence, Peyronie's disease, and altered anatomic integrity of the tunica albuginea) [2]. Neurogenic etiology encompasses lesions of the upper motor neuron, sacral lesions, or pudendal nerve injury. Finally, iatrogenic etiology is extended to pelvic surgery and drug intake (e.g., $\beta$-blockers, anti-androgens, and luteinizing hormone-releasing agonists and antagonists, etc.) [2].

\subsection{Epidemiology}

ED has a very high prevalence among the general population. A recent cross-sectional non-interventional study carried out on almost 10 million American men aged $>18$ years has reported that 573,313 men $(\sim 6 \%)$ have ED. The prevalence increases from the age of 18 to that of 59, while it decreases in men from 60 to 90 years [5]. Interestingly, patients with ED showed a significantly higher prevalence of cardiovascular disease (CVD) (18\% vs. $10 \%)$, DM (24\% vs. $11 \%$ ), and depression (11\% vs. $5 \%$ ) compared to patients without ED. The prevalence almost doubled in all these cases [5].

To better explore the association between DM and ED, other studies have assessed the prevalence of ED in patients with DM. A meta-analysis on 145 studies including almost 90,000 patients (mean age $55.8 \pm 7.9$ years) showed a prevalence of ED of $52.5 \%$ in patients with DM, after adjustment for publication bias [6]. Specifically, the prevalence was 37.5\% in type I DM (T1DM) and 66.3\% in type II DM (T2DM). Furthermore, the studies $(n=17)$ that used the Sexual Health Inventory for Men (SHIM) questionnaire to diagnose ED showed a prevalence of $82.7 \%$, thus suggesting an even higher prevalence of ED when an accurate diagnostic tool (more than a simple interview) is used [6]. Other evidence collected in patients with DM, reported an age-dependent prevalence of ED, which also correlates with the severity of DM [7].

This evidence strongly highlights the importance of including ED among the longterm complications of DM, and the crucial role of the diabetologist in the early diagnosis of ED in patients with DM.

\subsection{Pathogenesis}

As previously discussed, several factors are involved in the pathogenesis of ED. Among these, hypogonadism and vasculogenic etiology are of particular relevance in patients with DM. These aspects should be investigated in diabetic patients complaining of ED.

Hypogonadism is often present in patients with DM. A meta-analysis including 37 cross-sectional, longitudinal, or intervention studies, enrolling 1822 T2DM patients and 10,009 controls, reported significantly lower total T (TT) serum levels in patients compared to controls. The same results were found for free- $\mathrm{T}$ and sex-hormone-binding globulin (SHBG) levels. Hence, the risk for hypogonadism is greater in patients with DM than in non-diabetic patients [8]. The reason for this association relies on several pathogenetic 
mechanisms and, in particular, the insulin- and leptin-dependent altered function of the gonadotropin-releasing hormone $(\mathrm{GnRH})$ neuron. Accordingly, GnRH-neuron-secreting activity is influenced by low or high leptin or insulin levels. Insulin receptors are expressed in GnRH neurons but it is not entirely clear whether insulin influences the function of these neurons [9]. In contrast, leptin, released by the adipose tissue, stimulates kisspeptin secretion by the arcuate nucleus that, in turn, triggers GnRH secretion [10]. Patients without diabetes show an obesity-dependent prevalence of hypogonadism and about 33\% of obese men have hypogonadism [11]. Similarly, the prevalence of hypogonadism in patients with DM is influenced by the presence of obesity, reaching about $44 \%$ in these latter patients [11]. This is of particular relevance, as highlighted by the high prevalence of hypogonadism in patients with so-called "diabesity". Therefore, DM2 increases the prevalence of hypogonadism and the resulting low serum testosterone levels further worsen the health of diabetic patients. This further damages endothelial function since hypotestosteronemia reduces the expression and activity of NO synthase and increases asymmetric dimethylarginine (ADMA) expression [12]. ADMA levels correlate with some cardiovascular risk factors and inflammatory markers, so higher C-reactive protein (CRP) levels are commonly found in patients with DM2 and hypogonadism [13-15]. Finally, hypotestosteronemia modifies, with an androgen-receptor-mediated mechanism, the effects of insulin on the skeletal muscle by decreasing peroxisome-proliferator-activated receptor$\gamma$. This results in higher insulin resistance and worse glycolipid metabolism [16]. The 2020 Standards of Medical Care in Diabetes, released by the American Diabetes Association [17], suggest measuring serum TT levels in patients with DM and symptoms of hypogonadism (e.g., decreased sexual desire or ED). Therefore, diabetologists have a crucial role in the timely diagnosis of hypogonadism. Proper management of hypogonadism is important to improve glycolipid metabolism and cardiovascular health. Indeed, T deficiency has been associated with MACE and independently predicts in-stent restenosis among patients with acute coronary syndrome [18].

In addition to hypogonadism, chronic hyperglycemia activates alternative biochemical reactions, such as non-enzymatic glycation worsening endothelial function. Advanced endglycation products (AGEs) show direct toxicity as well as receptor-mediated mechanisms, likely involving MAPK-ERK/JNK pathways, in causing vascular damage induced by glycoxidation $[19,20]$.

Moreover, nervous and vasculogenic factors can play a role in the pathogenesis of ED in patients with DM. Microangiopathy causes distress to the vasa nervorum also involving the pudendal nerve that controls erection. A recent multicenter cross-sectional study has positively associated diabetic neuropathy with severe ED in patients older than 65 [21]. Macroangiopathy is an important long-term consequence of DM as well. Since the erection is based on a competent vascular mechanism, the presence of atherosclerotic plaques can lead to arterial ED. The importance of an early diagnosis of a vasculogenic etiology lies in its value as a marker of coronary artery disease, as the so-called "artery size hypothesis" suggests. According to this hypothesis, since the diameter of the penile arteries is smaller than that of the coronary arteries, arteriosclerosis will first show its effects in the smalldiameter arteries leading to ED, and subsequently, symptoms of coronary obstruction will follow [22]. Consequently, an early diagnosis of arterial ED can help diagnose the presence of subclinical coronary artery disease if patients are asked to undergo cardiac evaluation. In fact, a systematic review with a meta-analysis on 154,794 patients recently reported a significant increase in the risk of cardiovascular disease, coronary heart disease (CHD), and stroke in patients with ED compared with those without ED. The risk was 43\%, 59\%, and $34 \%$, respectively [23]. Similarly, a second systematic review with meta-analysis on longitudinal studies, recruiting 92,757 participants followed prospectively for an average of 6.1 years, concluded that there was a significantly higher risk of cardiovascular events in ED patients than in those without ED [24]. Finally, a meta-analysis of observational studies including 22,586 patients reported an odds ratio (OD) of 1.74 for CHD in patients with DM 
and ED compared to those with DM but without ED [25]. This suggests that the presence of ED further increases the risk for MACE in patients with DM.

\subsection{Diagnosis}

The diagnosis of ED starts with the patient's interview. When the anamnesis suggests the presence of ED, the administration of a validated questionnaire can be useful to objectively assess its presence and severity. In this regard, the International Index of Erectile Function (IIEF) represents a useful and validated tool [26]. In patients with DM referred for ED, the diagnostic flow-chart should include the evaluation of both TT serum levels and penile echo-color Doppler ultrasound (PCDU) examination after intracavernous injection (ICI).

According to the Endocrine Society, the diagnosis of hypogonadism can be made in the case of TT serum levels below the cut-off of $264 \mathrm{ng} / \mathrm{dL}(9.2 \mathrm{nmol} / \mathrm{L})$ in at least two different measurements [27]. However, it must be considered that DM has been listed among the conditions that lower either SHBG or TT below the normal range. In these cases, the clinician has to consider that a value of free $\mathrm{T}<64 \mathrm{pg} / \mathrm{mL}(220 \mathrm{pmol} / \mathrm{L})$ confirms the presence of hypogonadism [27].

PCDU examination after ICI with prostaglandin $(\mathrm{Pg}) \mathrm{E}_{1}$ or derivatives (e.g., alprostadil) is a second-line diagnostic test in patients with ED. Despite the lack of standardization of sampling location [28] and cut-off values, a major effort has been made over the decades to identify PCDU waveforms predicting arterial or venous ED [3]. The PCDU allows the measurement of the peak systolic velocity (PSV) and end-diastolic velocity (EDV) of the cavernous arteries in response to ICI drug administration (most often alprostadil). Both of these parameters describe the characteristics of blood flow in the cavernous arteries during erection and are currently used for the diagnosis of vasculogenic ED. Consequently, a restriction of the lumen of the cavernous artery (e.g., due to an arterial plaque or to a greater media-intima thickness) causes arterial blood flow to slow down and the PSV value decreases accordingly. In this regard, a PSV cut-off value between 25 and $35 \mathrm{~cm} / \mathrm{s}$ is used as the cut-off. Conversely, in the case of dysfunction of the veno-occlusive mechanism (blood is drained too quickly from the dorsal vein of the penis), the EDV increases $(>5 \mathrm{~cm} / \mathrm{s}$ ) [3]. In particular, a retrospective study of up to 300 patients with ED followed for 10 years reported that morphological abnormalities of the cavernous arteries (e.g., stenosis, atherosclerotic plaques, and intima-media thickness) were closely associated with cardiovascular disease. In fact, the risk for MACE was three times higher in patients with morphological abnormalities of the cavernous arteries than in those with normal morphology [29]. Once again, this evidence highlights the crucial importance of correct diagnosis and management of ED, given its consequences on cardiovascular health, especially in patients with DM.

\subsection{Therapeutic Options}

The therapeutic approach to ED differs according to its etiology. Hypogonadism can be overcome after weight loss in patients with "diabesity" [11]. Indeed, weight loss decreases serum levels of leptin, which increases the secretion of kisspeptin and the function of GnRH neurons, thus restoring hypothalamic-pituitary-gonadal function. Consequently, restoration of $\mathrm{T}$ levels to the normal range has been reported in obese and hypogonadal male patients after weight loss by prescribing a very-low-calorie ketogenic diet [30]. In the case of overt hypogonadism, testosterone replacement therapy (TRT) may be necessary [27]. In particular, an 11-year-long longitudinal study showed diabetes remission after long-term treatment with injectable $\mathrm{T}$ in patients with hypogonadism and T2DM [31].

There are several formulations available that contain testosterone. They include injectable ones consisting of testosterone undecanoate, propionate, phenyl-propionate, isocaproate, decanoate, or enanthate, or a transdermal gel containing testosterone. The oral formulation is little used in clinical practice due to the difficulty of maintaining stable values of serum testosterone due to the variability of its absorption through the intestinal tract. 
The choice of the best formulation for TRT depends on patient compliance, as available testosterone-containing available products offer daily, weekly, or monthly administration. Before starting TRT, the risk of prostate cancer and erythrocytosis should be considered. Furthermore, exogenous testosterone temporarily suppresses the hypothalamus-pituitarytestis axis and thus spermatogenesis and fertility. Therefore, TRT should not be prescribed to patients seeking paternity. Alternative treatment is based on the administration of human chorionic gonadotropin [32].

When the cause of ED is vasculogenic, correction of cardiovascular risk factors (e.g., hypertension, hypercholesterolemia, smoking, and obesity) is mandatory. After this step, prescribing phosphodiesterase type 5 inhibitors (PDE5i) is a useful choice. PDE5i acts by hindering the action of the PDE5 enzyme, which in turn increases the bioavailability of NO and cyclic GMP. Several molecules are currently available in clinical practice. These include sildenafil, tadalafil, vardenafil, and avanafil, which differ for bioavailability, pharmacokinetics, affinity, and selectivity for PDE isoenzymes. Sildenafil is mostly used on demand, due to its short bioavailability, while tadalafil can be used daily and could be preferred in patients with contemporary benign prostatic hyperplasia [33]. It is noteworthy that PDE5 is involved in the intracellular signaling of insulin. An in vitro study showed that insulin can stimulate glucose transport via the NO/cyclic GMP pathway in human vascular smooth cells [34]. Furthermore, sildenafil is capable of lowering insulin resistance in human endothelial cells [35]. These findings have led to studies that have assessed the effects of PDE5i on glycemic control in patients with T2DM. The meta-analysis of these studies concluded that sildenafil has no effect on glycated hemoglobin. However, only four studies were included, for a total of only 100 patients with T2DM and 98 controls that were treated with very different doses of sildenafil at various durations [36]. Hence, more evidence based on a greater number of patients and using proper study designs is necessary to better understand the role of PDE5i in the glycometabolic control in patients with DM. Moreover, the benefits of PDE5is have been reported in diabetic cardiopathy, where these molecules can decrease the pro-inflammatory chemokine interleukin-8 [37]. This further indicates the need for a better investigation of the role of PDE5i in patients with DM and ED. Pharmacotherapy also includes local application of alprostadil that can be administered as a topical cream or medicated pellet, both adsorbed via the urethral meatus [38].

Other treatments include vacuum devices, whose efficacy is high but with a slightly increased risk of minor (pain) and major (skin necrosis) adverse events [38,39]. Lowintensity extracorporeal shock wave therapy has been shown to be effective in treating ED by improving the IIEF score and PSV value on the PCDU evaluation [40]. Finally, surgical treatment with inflatable or semi-rigid prosthesis implantation is available for patients who are not responsive to pharmacotherapy or who want a definitive resolution to the problem [38].

\section{Conclusions}

$\mathrm{DM}$ is a chronic and widespread disease whose prevalence is expected to increase in the coming years [1]. Updated knowledge of the complications of this disease is of great importance. However, if some complications are well coded and followed appropriately, sexual dysfunctions and especially ED are not. Nonetheless, ED is a very common complication in male patients with DM. Furthermore, its prevalence increases with advancing age and in patients with long-lasting diabetes and of greater severity, reflecting worse glycometabolic control and worse cardiovascular health. Therefore, an early and timely diagnosis of ED is important to detect the presence of hypogonadism and/or CHD. Correction of cardiovascular risk factors is mandatory in DM patients with ED. Moreover, PDE5is are a useful therapeutic choice, and further studies are warranted to better understand the role of these drugs, if any, in glucose control and cardiovascular health. Taking all these aspects into account, ED can be considered a long-term complication of DM and, 
in this view, the diabetologist has a pivotal role in its early detection and in the correct management of this aspect (Figure 1).

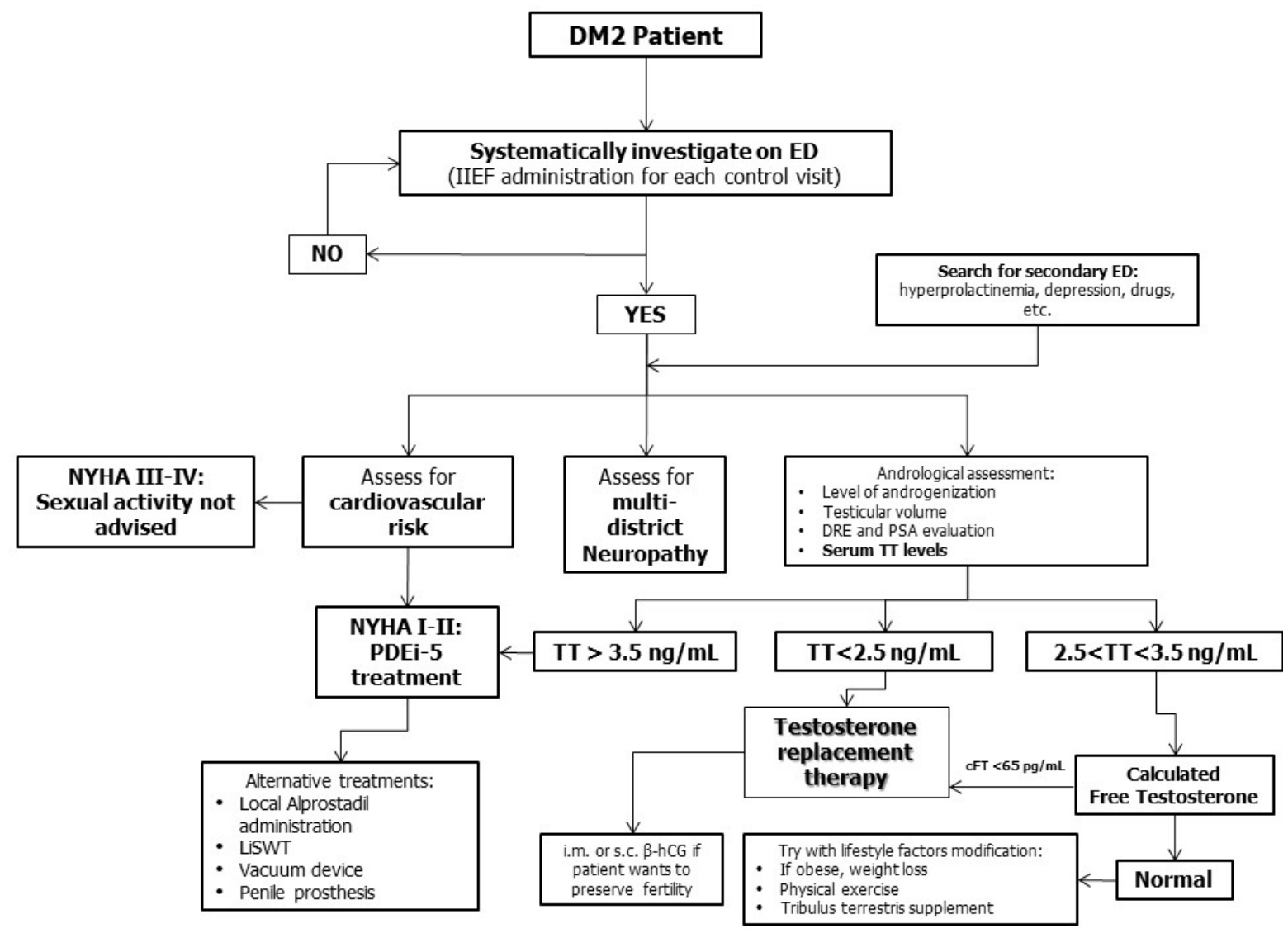

Figure 1. Diagnostic and therapeutic algorithm of erectile dysfunction (ED) management in patients with diabetes mellitus (DM). A systematic evaluation for ED should be carried out in patients with DM. Diagnostic assessment should include evaluation of cardiovascular risk, multidistrict neuropathy, testicular function, and secondary ED. Sexual activity is not recommended in patients with NYHA class III-IV, while treatment can be prescribed in those in NYHA class I-II. Testosterone replacement treatment is indicated in patients with hypogonadism having serum total testosterone (TT) levels consistently below $2.5 \mathrm{ng} / \mathrm{mL}$ or between 2.5 and $3.5 \mathrm{ng} / \mathrm{mL}$ and low calculated free testosterone.

Author Contributions: Conceptualization, R.C.; methodology, F.B.; data curation, R.A.C. and S.L.V.; writing-original draft preparation, R.C., A.C. and C.G.; writing-review and editing, A.E.C.; supervision, A.E.C. All authors have read and agreed to the published version of the manuscript.

Funding: This research did not receive any specific grant from any funding agency in the public, commercial, or not-for-profit sector.

Conflicts of Interest: The authors declare that there are no conflicts of interest that could be perceived as prejudicing the impartiality of the research reported.

\section{Abbreviations}

DRE: digital-rectal exploration; hCG, human chorionic gonadotropin; IIEF-5, International Index of Erectile Function; LiSWT, low intensity shock wave therapy; NYHA, New York Heart Association, PDE5i, phosphodiesterase 5 inhibitors.

\section{References}

1. NIH Consensus Conference. Impotence. NIH Consensus Development Panel on Impotence. JAMA 1993, 270, 83-90. [CrossRef]

2. Calogero, A.E.; Burgio, G.; Condorelli, R.A.; Cannarella, R.; La Vignera, S. Epidemiology and risk factors of lower urinary tract symptoms/benign prostatic hyperplasia and erectile dysfunction. Aging Male 2018, 22, 12-19. [CrossRef] [PubMed] 
3. Zvara, P.; Sioufi, R.; Schipper, H.M.; Begin, L.R.; Brock, G.B. Nitric oxide mediated erectile activity is a testosterone dependent event: A rat erection model. Int. J. Impot. Res. 1995, 70, 209-219.

4. Cannarella, R.; Calogero, A.E.; Aversa, A.; Condorelli, R.A.; La Vignera, S. Differences in Penile Hemodynamic Profiles in Patients with Erectile Dysfunction and Anxiety. J. Clin. Med. 2021, 10, 402. [CrossRef] [PubMed]

5. Goldstein, I.; Chambers, R.; Tang, W.; Stecher, V.; Hassan, T. Real-world observational results from a database of 48 million men in the United States: Relationship of cardiovascular disease, diabetes mellitus and depression with age and erectile dysfunction. Int. J. Clin. Pract. 2018, 72, e13078. [CrossRef]

6. Kouidrat, Y.; Pizzol, D.; Cosco, T.; Thompson, T.; Carnaghi, M.; Bertoldo, A.; Solmi, M.; Stubbs, B.; Veronese, N. High prevalence of erectile dysfunction in diabetes: A systematic review and meta-analysis of 145 studies. Diabet. Med. 2017, 34, 1185-1192. [CrossRef]

7. Klein, R.; E Klein, B.; E Lee, K.; E Moss, S.; Cruickshanks, K.J. Prevalence of self-reported erectile dysfunction in people with long-term IDDM. Diabetes Care 1996, 19, 135-141. [CrossRef]

8. Corona, G.; Monami, M.; Rastrelli, G.; Aversa, A.; Sforza, A.; Lenzi, A.; Forti, G.; Mannucci, E.; Maggi, M. Type 2 diabetes mellitus and testosterone: A meta-analysis study. Int. J. Androl. 2010, 34, 528-540. [CrossRef]

9. Evans, M.C.; Hill, J.W.; Anderson, G.M. Role of insulin in the neuroendocrine control of reproduction. J. Neuroendocr. 2021, 33, e12930. [CrossRef]

10. Childs, G.V.; Odle, A.K.; MacNicol, M.C.; MacNicol, A.M. The Importance of Leptin to Reproduction. Endocrinology $2020,162$. [CrossRef]

11. Dhindsa, S.; Ghanim, H.; Batra, M.; Dandona, P. Hypogonadotropic Hypogonadism in Men with Diabesity. Diabetes Care 2018, 41, 1516-1525. [CrossRef]

12. Tariq, K.; Khan, M.A. Asymmetrical dimethyl arginine in type 2 diabetic patients with coronary artery disease. J. Pak. Med. Assoc. 2016, 66, 957-960. [PubMed]

13. Wierzbicki, A.S.; Solomon, H.; Lumb, P.J.; Lyttle, K.; Lambert-Hammill, M.; Jackson, G. Asymmetric dimethyl arginine levels correlate with cardiovascular risk factors in patients with erectile dysfunction. Atherosclerosis 2006, 185, 421-425. [CrossRef]

14. Stühlinger, M.C.; Stanger, O. Asymmetric dimethyl-L-arginine (ADMA): A possible link between homocyst(e)ine and endothelial dysfunction. Curr. Drug Metab. 2005, 6, 3-14. [CrossRef] [PubMed]

15. Jamwal, S.; Sharma, S. Vascular endothelium dysfunction: A conservative target in metabolic disorders. Inflamm. Res. 2018, 67, 391-405. [CrossRef]

16. Rao, P.M.; Kelly, D.M.; Jones, T.H. Testosterone and insulin resistance in the metabolic syndrome and T2DM in men. Nat. Rev. Endocrinol. 2013, 9, 479-493. [CrossRef] [PubMed]

17. Table of Contents. Available online: https://care.diabetesjournals.org/content/43/Supplement_1 (accessed on 30 July 2021).

18. Chmiel, A.; Mizia-Stec, K.; Wierzbicka-Chmiel, J.; Rychlik, S.; Muras, A.; Mizia, M.; Bienkowski, J. Low testosterone and sexual symptoms in men with acute coronary syndrome can be used to predict major adverse cardiovascular events during long-term follow-up. Andrology 2015, 3, 1113-1118. [CrossRef]

19. Beisswenger, P.J. Glycation and biomarkers of vascular complications of diabetes. Amino Acids 2010, 42, 1171-1183. [CrossRef]

20. De Nigris, F.; Rienzo, M.; Sessa, M.; Infante, T.; Cesario, E.; Ignarro, L.J.; Al-Omran, M.; Giordano, A.; Palinski, W.; Napoli, C. Glycoxydation promotes vascular damage via MAPK-ERK/JNK pathways. J. Cell Physiol. 2012, 227, 3639-3647. [CrossRef]

21. Furukawa, S.; Sakai, T.; Niiya, T.; Miyaoka, H.; Miyake, T.; Yamamoto, S.; Maruyama, K.; Ueda, T.; Senba, H.; Todo, Y.; et al. Diabetic peripheral neuropathy and prevalence of erectile dysfunction in Japanese patients aged $<65$ years with type 2 diabetes mellitus: The Dogo Study. Int. J. Impot. Res. 2016, 29, 30-34. [CrossRef] [PubMed]

22. Montorsi, P.; Ravagnani, P.M.; Galli, S.; Rotatori, F.; Briganti, A.; Salonia, A.; Rigatti, P.; Montorsi, F. The Artery Size Hypothesis: A Macrovascular Link between Erectile Dysfunction and Coronary Artery Disease. Am. J. Cardiol. 2005, 96, 19-23. [CrossRef]

23. Zhao, B.; Hong, Z.; Wei, Y.; Yu, D.; Xu, J.; Zhang, W. Erectile Dysfunction Predicts Cardiovascular Events as an Independent Risk Factor: A Systematic Review and Meta-Analysis. J. Sex. Med. 2019, 16, 1005-1017. [CrossRef] [PubMed]

24. Vlachopoulos, C.; Terentes-Printzios, D.; Ioakeimidis, N.; Aznaouridis, K.; Rokkas, K.; Synodinos, A.; Christoforatou, E.; Aggelis, A.; Samentzas, A.; Stefanadis, C. Prediction of cardiovascular events and all-cause mortality with erectile dysfunction: A systematic review and meta-analysis of cohort studies. J. Am. Coll. Cardiol. 2012, 59, E2074. [CrossRef]

25. Yamada, T.; Hara, K.; Umematsu, H.; Suzuki, R.; Kadowaki, T. Erectile Dysfunction and Cardiovascular Events in Diabetic Men: A Meta-analysis of Observational Studies. PLoS ONE 2012, 7, e43673. [CrossRef] [PubMed]

26. Rosen, R.C.; Cappelleri, J.C.; Gendrano, N. The International Index of Erectile Function (IIEF): A state-of-the-science review. Int. J. Impot. Res. 2002, 14, 226-244. [CrossRef]

27. Bhasin, S.; Brito, J.P.; Cunningham, G.R.; Hayes, F.J.; Hodis, H.N.; Matsumoto, A.M.; Snyder, P.J.; Swerdloff, R.S.; Wu, F.C.; A Yialamas, M. Testosterone Therapy in Men with Hypogonadism: An Endocrine Society* Clinical Practice Guideline. J. Clin. Endocrinol. Metab. 2018, 103, 1715-1744. [CrossRef] [PubMed]

28. Pagano, M.; Stahl, P.J. Variation in Penile Hemodynamics by Anatomic Location of Cavernosal Artery Imaging in Penile Duplex Doppler Ultrasound. J. Sex. Med. 2015, 12, 1911-1919. [CrossRef]

29. Caretta, N.; Ponce, M.D.R.; Minicuci, N.; Palego, P.; Valente, U.; Garolla, A.; Ferlin, A.; Foresta, C. Penile doppler ultrasound predicts cardiovascular events in men with erectile dysfunction. Andrology 2018, 7, 82-87. [CrossRef] [PubMed] 
30. La Vignera, S.; Cannarella, R.; Galvano, F.; Grillo, A.; Aversa, A.; Cimino, L.; Magagnini, C.M.; Mongioì, L.M.; Condorelli, R.A.; Calogero, A.E. The ketogenic diet corrects metabolic hypogonadism and preserves pancreatic B-cell function in overweight/obese men: A single-arm uncontrolled study. Endocrine 2020. [CrossRef]

31. Haider, K.S.; Haider, A.; Saad, F.; Doros, G.; Hanefeld, M.; Dhindsa, S.; Dandona, P.; Traish, A. Remission of type 2 diabetes following long-term treatment with injectable testosterone undecanoate in patients with hypogonadism and type 2 diabetes: 11-year data from a real-world registry study. Diabetes, Obes. Metab. 2020, 22, 2055-2068. [CrossRef]

32. Corona, G.; Goulis, D.G.; Huhtaniemi, I.; Zitzmann, M.; Toppari, J.; Forti, G.; Vanderschueren, D.; Wu, F.C. European Academy of Andrology (EAA) guidelines on investigation, treatment and monitoring of functional hypogonadism in males: Endorsing organization: European Society of Endocrinology. Andrology 2020, 8, 970-987. [CrossRef]

33. Sebastianelli, A.; Spatafora, P.; Morselli, S.; Vignozzi, L.; Serni, S.; McVary, K.T.; Kaplan, S.; Gravas, S.; Chapple, C.; Gacci, M. Tadalafil Alone or in Combination with Tamsulosin for the Management for LUTS/BPH and ED. Curr. Urol. Rep. 2020, $21,1-12$. [CrossRef]

34. Bergandi, L.; Silvagno, F.; Russo, I.; Riganti, C.; Anfossi, G.; Aldieri, E.; Ghigo, D.; Trovati, M.; Bosia, A. Insulin Stimulates Glucose Transport Via Nitric Oxide/Cyclic GMP Pathway in Human Vascular Smooth Muscle Cells. Arterioscler. Thromb. Vasc. Biol. 2003, 23, 2215-2221. [CrossRef] [PubMed]

35. Mammi, C.; Pastore, D.; Lombardo, M.F.; Ferrelli, F.; Caprio, M.; Consoli, C.; Tesauro, M.; Gatta, L.; Fini, M.; Federici, M.; et al. Sildenafil Reduces Insulin-Resistance in Human Endothelial Cells. PLoS ONE 2011, 6, e14542. [CrossRef]

36. Poolsup, N.; Suksomboon, N.; Aung, N. Effect of phosphodiesterase-5 inhibitors on glycemic control in person with type 2 diabetes mellitus: A systematic review and meta-analysis. J. Clin. Transl. Endocrinol. 2016, 6, 50-55. [CrossRef] [PubMed]

37. Giannattasio, S.; Corinaldesi, C.; Colletti, M.; Di Luigi, L.; Antinozzi, C.; Filardi, T.; Scolletta, S.; Basili, S.; Lenzi, A.; Morano, S.; et al. The phosphodiesterase 5 inhibitor sildenafil decreases the proinflammatory chemokine IL-8 in diabetic cardiomyopathy: In vivo and in vitro evidence. J. Endocrinol. Investig. 2018, 42, 715-725. [CrossRef]

38. Salonia, A.; Bettocchi, C.; Boeri, L.; Capogrosso, P.; Carvalho, J.; Cilesiz, N.C.; Cocci, A.; Corona, G.; Dimitropolous, K.; Gül, M.; et al. EAU Working Group on Male Sexual and Reproductive Health. European Association of Urology Guidelines on Sexual and Re-productive Health-2021 Update: Male Sexual Dysfunction. Eur. Urol. 2021, 25, S0302-2838(21)01813-3.

39. Beaudreau, S.A.; Van Moorleghem, K.; Dodd, S.M.; Liou-Johnson, V.; Suresh, M.; Gould, C.E. Satisfaction with a Vacuum Constriction Device for Erectile Dysfunction among Middle-Aged and Older Veterans. Clin. Gerontol. 2020, 44, 307-315. [CrossRef]

40. Sokolakis, I.; Hatzichristodoulou, G. Clinical studies on low intensity extracorporeal shockwave therapy for erectile dysfunction: A systematic review and meta-analysis of randomised controlled trials. Int. J. Impot. Res. 2019, 31, 177-194. [CrossRef] [PubMed] 